OPEN ACCESS

Edited by:

Nilima Prakash,

Hamm-Lippstadt University of Applied

Sciences, Germany

Reviewed by:

Laure Bally-Cuif,

Institut Pasteur, France

Ralf Kühn,

Max Delbrück Center for Molecular

Medicine (HZ), Germany

*Correspondence:

Michael Brand

michael.brand@biotec.tu-dresden.de

Received: 28 April 2017

Accepted: 19 June 2017

Published: 30 June 2017

Citation:

Kesavan G, Chekuru A, Machate A and Brand $M$ (2017)

CRISPR/Cas9-Mediated Zebrafish Knock-in as a Novel Strategy to Study

Midbrain-Hindbrain Boundary

Development.

Front. Neuroanat. 11:52

doi: 10.3389/fnana.2017.00052

\section{CRISPR/Cas9-Mediated Zebrafish Knock-in as a Novel Strategy to Study Midbrain-Hindbrain Boundary Development}

\author{
Gokul Kesavan, Avinash Chekuru, Anja Machate and Michael Brand * \\ Biotechnology Center and DFG-Research Center for Regenerative Therapies Dresden, Technische Universität Dresden, \\ Dresden, Germany
}

The midbrain-hindbrain boundary $(\mathrm{MHB})$ acts as an organizer and controls the fate of neighboring cells to develop into either mesencephalic (midbrain) or metencephalic (hindbrain) cells by secreting signaling molecules like Wnt1 and Fgf8. The zebrafish is an excellent vertebrate model for studying MHB development due to the ease of gene manipulation and the possibility of following cellular dynamics and morphogenetic processes using live imaging. Currently, only very few reporter and/or Cre-driver lines are available to study gene expression at the MHB, hampering the understanding of $\mathrm{MHB}$ development, and traditional transgenic technologies using promoter/enhancer fragments or bacterial artificial chromosome (BAC)-mediated transgenesis often do not faithfully recapitulate endogenous expression patterns. In contrast, CRISPR/Cas9-mediated genome editing technology now provides a great opportunity to efficiently knock-in or knock-out genes. We have generated four CRISPR/Cas9-based knock-in fluorescent reporter lines for two crucial genes involved in MHB development, namely otx2 and pax2a. The coding sequences of the reporters were knocked-in upstream of the corresponding ATG and are, thus, under the control of the endogenous promoter/enhancer elements. Interestingly, this strategy does not disturb endogenous gene expression. Using the fast maturing fluorescent protein reporter, Venus, enabled us to follow MHB development using cell tracking and live imaging. In addition, we show that these reporter lines label various neuronal and glial cell types in the adult zebrafish brain, making them highly suitable for investigating embryonic and adult midbrain, hindbrain, and MHB development.

Keywords: CRISPR/Cas9, midbrain-hindbrain boundary (MHB), knock-in reporter, zebrafish and transgenesis

\section{INTRODUCTION}

The boundary between the midbrain (mesencephalon, mes) and the hindbrain (metencephalon, met) is essential for establishing a clear demarcation between the midbrain and the anterior hindbrain. This midbrain-hindbrain boundary (MHB), also known as the isthmic organizer or midbrain-hindbrain organizer, acts as a local signaling center (Wurst and Bally-Cuif, 2001; Raible and Brand, 2004; Rhinn et al., 2006; Dworkin and Jane, 2013). The MHB forms at the interface of two transcription factor domains in the neural plate epithelium, namely the anterior Otx 
domain and the posterior Gbx domain (Rhinn et al., 2003). This interface is established by mutual transcriptional repression and initiates formation of the prospective MHB. Successively, morphogens such as Wnt, Fgf, and transcription factors like Engrailed $1 / 2$, and Pax2/5/8 induce the formation of the MHB, and their subsequent interplay is critical for maintenance of the MHB (Rhinn and Brand, 2001; Wurst and Bally-Cuif, 2001; Raible and Brand, 2004; Rhinn et al., 2006; Dworkin and Jane, 2013). The above-mentioned factors (Otx, Gbx, Wnt, Fgf8, Pax, and Eng) form the core of the MHB signaling machinery and a disruption of any of these factors interferes with the formation and function of the MHB.

Several model organisms, including chicken, mouse, and zebrafish, have been extensively used to understand the complex network of genes and their interactions during MHB development (Martinez-Barbera et al., 2001; Raible and Brand, 2004; Zervas et al., 2004; Rhinn et al., 2006; Sunmonu et al., 2011; Tossell et al., 2011). The zebrafish model has been instrumental in the identification of several genes that are essential for MHB development due to the availability of numerous mutants obtained from large-scale mutagenesis screens (Brand et al., 1996; Schier et al., 1996).

The zebrafish is an ideal model to study vertebrate brain development, and especially patterning in central nervous system, beginning from the neural plate stages because of the following reasons. (A) Fertilization is external and a single female can produce several hundred eggs $(>200)$ in a single spawning; (B) the transparent nature of the embryos allows real-time imaging of the developing embryos; (C) fast embryonic development-a fertilized egg develops into a larva with most organs fully formed within 3 days; and (D) the possibility of manipulating genes and the ease of performing both loss- and gain-of-function experiments. Besides neurobiology studies in the embryo and larva, the adult zebrafish brain has widespread proliferative neural stem cells (neurogenic zones) distributed along the rostro-caudal axis (Adolf et al., 2006; Grandel et al., 2006). The zebrafish has also become a valuable model system for understanding neural stem cell heterogeneity, adult neurogenesis under homeostasis and injury, and regeneration (reviewed in Kizil et al., 2012; Grandel and Brand, 2013; Alunni and Bally-Cuif, 2016). Therefore, the ability to combine genetic manipulations with the application of advanced microscopic techniques, both in developing and adult animals, makes zebrafish a unique model to study vertebrate neurogenesis.

Effective study of adult neurogenesis or regeneration often requires labeling of specific cell types such as stem cells or neurons. The midbrain tectum, with its stratified cellular organization, is not only structurally similar to the mammalian cortex but is also involved in visual input processing and coordination of goal directed movements. Thus, it collectively acts as a major visual processing center in the brain. To successfully understand visual processing mechanisms, it is imperative to label individual cells or a group of cells in a neuronal network (Robles et al., 2011), and a majority of the previous studies mostly relied on neuroanatomical studies in other teleosts for such insight (Meek and Schellart, 1978; Meek, 1981). Nevertheless, few studies were successful in labeling cell populations of the midbrain tectum using Tol2-based transgenesis or gene-trap based random integration methods (Scott and Baier, 2009; Muto et al., 2013). Until recently, genetic manipulations in the zebrafish mainly entailed constitutive mutants generated using random mutagenesis screens or transposon-mediated random integration of transgenic constructs. Although, cellspecific promoters of interest would still be active in the adult fish, random integration often results in ectopic expression and/or gene silencing, thereby limiting the ability to consistently label specific cell types, especially in adult tissues. Thus, new genetic tools that reliably label cells by inserting reporter constructs at their native promoter sites would be of great interest.

Recent advances in genome editing that utilize sequencespecific DNA nucleases like Zinc finger nucleases, TALEN, and more importantly CRISPR/Cas9, have opened hitherto non-existent opportunities to knock-out or knock-in genes at precise locations in the zebrafish genome (Jao et al., 2013; Auer et al., 2014; Hoshijima et al., 2016). Double stranded breaks (DSB) created by the above-mentioned systems trigger cell repair mechanisms like the non-homologous end-joining (NHEJ) pathway that result in site-specific insertion/deletion (indel) mutations in the corresponding target sites. Recently, Auer et al. (2014) showed that reporter constructs could be efficiently integrated at the target site (TS) by providing a bait plasmid containing the TS, and have used this homologyindependent knock-in system to convert GFP reporter lines into Gal4 driver lines or to directly knock-in Gal4 transgenes. Adopting this strategy, we have generated knock-in reporters by targeting them to a $5^{\prime}$ sequence upstream of the ATG for some of the essential genes involved in MHB development, namely, Otx2 and pax2a. These transgenic lines act as read-outs for gene promoter activity and provide new tools to observe MHB development in real time.

\section{MATERIALS AND METHODS}

\section{Zebrafish Strains and Maintenance}

Zebrafish (Danio rerio) embryos were obtained by natural spawning. Both embryos and adults were raised and maintained at $28.5^{\circ} \mathrm{C}$ with a 14 -h light and 10 -h dark cycle (Westerfield, 2000; Brand et al., 2002). Embryos were staged as hours post fertilization (hpf) as described previously (Kimmel et al., 1995). The wild type strain $A B$ was used to generate reporter knock-in lines, and transgenic fish lines were maintained as outcrosses. Neither the larvae nor the adult fish from the generated reporter lines showed any physiological or behavioral abnormalities.

This study was carried out in accordance with the animal welfare law (Tierschutzgesetz, Federal republic of Germany) and the local authority (Landesdirektion Sachsen). Protocols for the generation (24-9168.11-1/2013-14) and maintenance of transgenic animals (DD24-5131/346/11 and DD24-5131/346/12) were appropriately approved (Landesdirektion Sachsen). Experimental animals were used according to the approved protocols (24-9168.24-1/2014-4). 


\section{Molecular Cloning}

Genomic DNA from the wild type strain $\mathrm{AB}$ was used to amplify bait sequences by PCR (Phusion Polymerase, Thermo Fischer) using primers listed in the Supplementary Table S2. To generate donor plasmids, baits were cloned into a pCS2+ or Topo PCR II vectors (Invitrogen) containing the coding sequence for the Venus fluorescent protein or turboRFP (promoterless tRFP plasmid, Evrogen). The CMV promoter was later removed from the $\mathrm{pCS} 2+$ vector. All constructs were verified by sequencing.

\section{Single-Guide RNA (sgRNA) Design and Off Targets}

The sgRNA sequences targeting the NGG-PAM motif were identified at about 500 base pairs upstream of the ATG start codon for a specific gene of interest. CHOP-CHOP web tool was used for selecting target sites (Montague et al., 2014). The sgRNA designed for ot 2 had the sequence GGAACccggCTA ATTGTCTCAGG while that for Pax2a was GGGGggatctGG GAAGGAGGGGG; the PAM sequences are underlined. Loss of the restriction site was used to estimate the efficiency of the sgRNA (HpaII and XhoII respectively, marked in lowercase). No
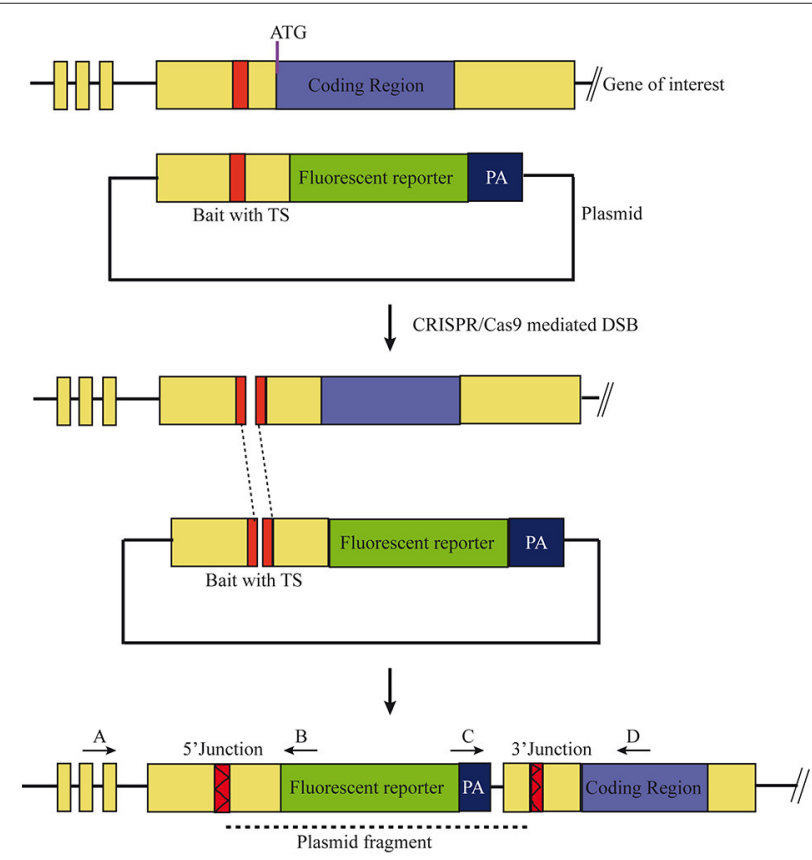

FIGURE 1 | Scheme of the knock-in strategy. A target site (TS), located 500 base pairs upstream of the ATG in the non-coding region in the gene of interest were chosen. The bait plasmid was constructed by cloning $1 \mathrm{~kb}$ of the upstream sequence, including the target site, into a plasmid with the desired fluorescent reporter and poly A (PA) sequence. The bait plasmid, sgRNA against the target site, and Cas 9 mRNA were injected at the 1-cell stage. The Cas9 protein creates double stranded breaks (DSB) at both TS, i.e., genomic locus and bait plasmid, and the linearized plasmid bait is integrated by homology independent repair. Forward integration of bait plasmid will result in expression of the fluorescent reporter that matches the expression pattern of the gene of interest. Primer pairs $(A+B, C+D)$ can be used to verify the $5^{\prime}$ and $3^{\prime}$ junctions of the knock-in, respectively. off-targets were identified for the chosen TS, using CHOP-CHOP i.e., there were no genomic targets with 2 bp mismatches (Cong et al., 2013). Further, the transgenic reporter lines have been outcrossed for more than 6 generations to date, thereby diluting out potential off target mutations.

\section{sgRNA, Cas9 Generation, and Injection into Zebrafish Embryos}

The sgRNA and Cas9 mRNA were prepared as previously described (Jao et al., 2013), and plasmids for generating mRNA were a gift from the Chen and Wente labs (sourced from addgene). All injections were carried out in the wild-type $A B$ strain embryo at the 1-cell stage. Each embryo was injected with a $1 \mathrm{nl}$ solution containing $35 \mathrm{ng} / \mu \mathrm{l}$ of sgRNA, $150 \mathrm{ng} / \mu \mathrm{l}$ of Cas $9 \mathrm{mRNA}$, and $25 \mathrm{ng} / \mu \mathrm{l}$ circular donor plasmid. The sgRNA and bait plasmid concentrations were optimized such that at least $50 \%$ of the injected embryos survived and showed normal development at $24 \mathrm{hpf}$. The injected embryos were monitored for the next 5 days and about 100 embryos were raised to adulthood for each transgenic line.

\section{Identification of Founders and Genotyping}

Injected embryos were raised and outcrossed with the wild type strains WIK or TL. Founders were identified by screening F1 embryos for the presence of a fluorescence signal at $24 \mathrm{hpf}$. The screening was stopped when 2 founders were identified for
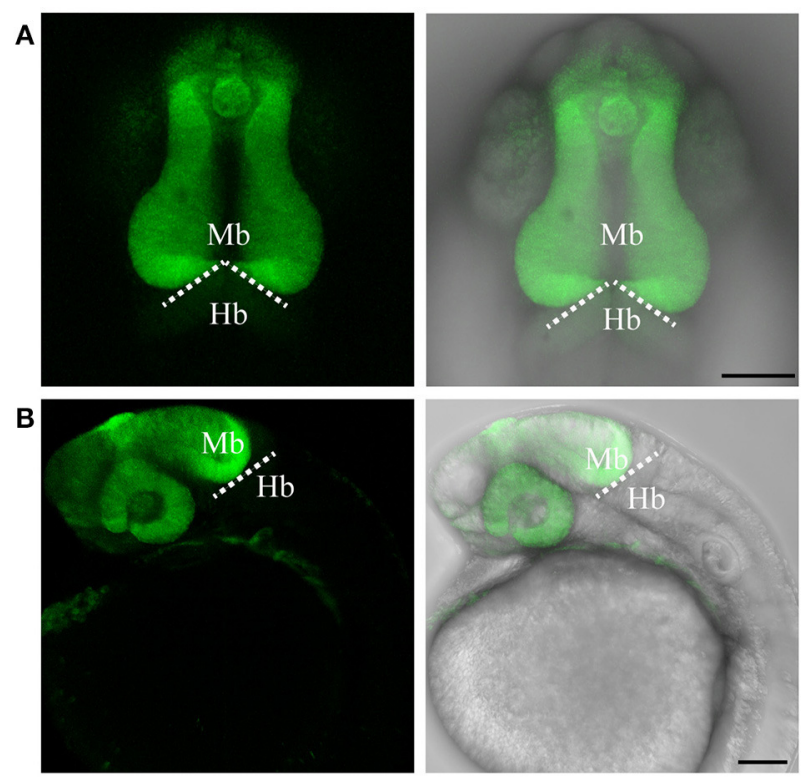

FIGURE 2 | Targeted knock-in of Venus fluorescent protein into the otx2 locus. Expression of Venus fluorescent protein at $24 \mathrm{hpf}$. Images were taken from live embryos anesthetized in MS-222. (A) Left panel shows dorsal view with the anterior of the embryo facing upwards, right panel is a merged image of the fluorescent and transmitted light channels. Venus is expressed in the retina and midbrain, sharply abutting the MHB (dotted line). (B) Lateral view of an embryo expressing Venus. Left panel shows fluorescent channel and right panel shows a merged image of the fluorescent and transmitted light channels. All images are maximum intensity projections covering $50 \mu \mathrm{m}$ tissue with a Z-interval of $2 \mu \mathrm{m}$. Mb, midbrain; Hb, hindbrain. Scale bar: $100 \mu \mathrm{m}$. 
each transgenic line. Genomic DNA was isolated from individual F1 embryos, and PCR amplification and subsequent sequencing were used to verify integration at the $5^{\prime}$ and $3^{\prime}$ junctions. F1 embryos with a fluorescence signal were raised and outcrossed to different wild-type strains (AB, WIK, or TL) between generations to reduce the general effects of inbreeding.

\section{RNA Extraction and Quantitative Real Time PCR (qRT PCR)}

For both otx2 and pax $2 a$, the Venus knock-in fish were crossed with the tRFP knock-in line and the double-positive (Venus ${ }^{+}$ and $\mathrm{tRFP}^{+}$) embryos were sorted at $48 \mathrm{hpf}$. Respective wildtype control embryos (double-negative) were also collected at 48 hpf. The embryos were pooled $(n=15)$, lysed in extrazol (BLIRT S.A.), RNA extracted, and treated with DNAse. One-step real time reverse transcription PCR (Takara) was performed on biological $(n=3)$ and technical $(n=3)$ replicates to quantify expression of otx 2 and pax $2 a$ in the double-positive transgenic embryos (Venus ${ }^{+}$and $\mathrm{tRFP}^{+}$) and was compared with that of wild-type embryos. Beta-actin was used as a house keeping gene to normalize the expression values. Fold changes were calculated using the $2^{-\Delta \Delta C_{T}}$ Method (Livak and Schmittgen, 2001) and the two tailed, unpaired " $t$ "-test was used to calculate statistical significance at a " $p$ "-value of 0.05 (Graph pad prism, ver. 5.0).
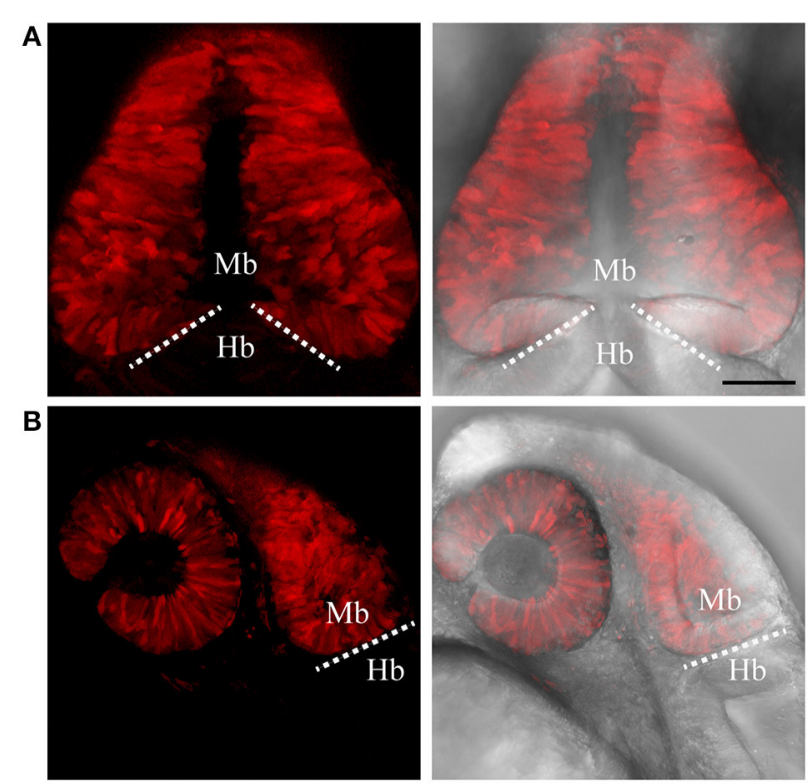

FIGURE 3 | Targeted knock-in of tRFP into the otx2 locus. Expression of tRFP at 24 hpf. Images were taken from live embryos anesthetized in MS-222. (A) Left panel shows a dorsal view with the anterior of the embryo facing upwards, right panel shows a merged image of fluorescent and transmitted light channels. tRFP is expressed in the retina and midbrain, sharply abutting the MHB (dotted line). (B) Lateral view of embryos expressing tRFP. Left panel shows fluorescent channel and right panel shows a merged image of the fluorescent and transmitted light channels. Images are maximum intensity projections covering $50 \mu \mathrm{m}$ tissue with a Z-interval of $2 \mu \mathrm{m}$. MB, midbrain; $\mathrm{Hb}$, hindbrain. Scale bar: $100 \mu \mathrm{m}$

\section{Tissue Preparation}

Embryos (24 hpf) were fixed with $4 \%$ paraformaldehyde (PFA) and stored in $100 \%$ methanol at $-20^{\circ} \mathrm{C}$. For adult fish, fish aged between 6 and 8 months were killed by an MS-222 overdose and the heads harvested after carefully removing the skull roof. Fish heads were fixed overnight in freshly prepared $4 \%$ PFA in $0.1 \mathrm{M}$ phosphate buffer $(\mathrm{PB}), \mathrm{pH}$ 7.4. Fixed samples were subjected to decalcification overnight in $\mathrm{PB}$ containing $0.5 \mathrm{M}$ EDTA and 20\% sucrose prior to embedding in $7.5 \%$ gelatin and $20 \%$ sucrose in PB. Next, fish heads were instantly frozen on dry ice and cryo-sectioned at 7-10 $\mu \mathrm{m}$ thicknesses on a Microm HM 560 cryostat. Cryopreserved heads were stored at $-80^{\circ} \mathrm{C}$ and the cryo-sectioned slides were stored at $-20^{\circ} \mathrm{C}$ for subsequent immunohistochemistry (IHC).

\section{In situ Hybridization}

Embryos (24 hpf) were fixed in 4\% PFA and stored in $100 \%$ methanol at $-20^{\circ} \mathrm{C}$. Whole mount in situ hybridization was performed as previously described (Reifers et al., 1998). Briefly, digoxigenin (DIG) or fluorescein-labeled probes, synthesized from linear DNA using a RNA labeling and detection kit (Roche), and hybridized probes were detected using anti-digoxigenin or anti-fluorescein antibodies. Antibody staining was visualized using BM purple (digoxigenin) or fast red (fluorescein). The stained embryos were dissected with sharpened tungsten needles,

A

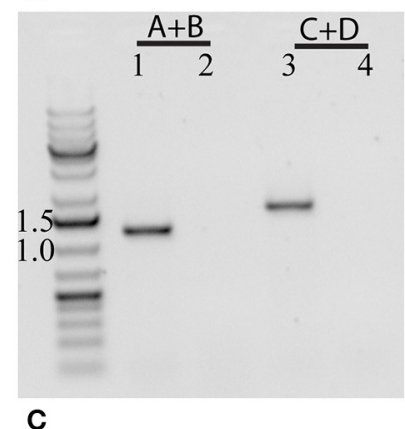

B

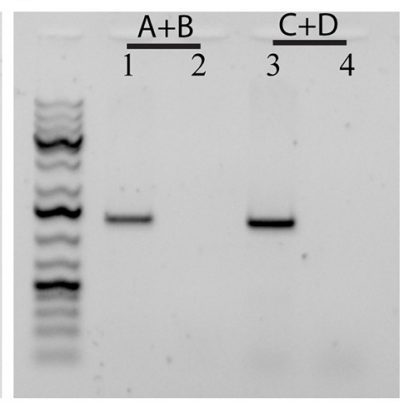

otx2:venus $\begin{array}{lll}\text { Wt } & \text { GGAACCCGGCTAATTGTCTCAGG } \\ (\mathrm{A}+\mathrm{B}) & \text { GGAACCCGGCTAATT } & \text { CAGG }(-4) \\ (\mathrm{C}+\mathrm{D}) & \text { GGAACCCGGCTAATT } & \text { CAGG }(+13)\end{array}$

GTGAATACCGGCT

otx2:tRFP

\section{GGAACCCGGCTAATTGTCTCAGG $(\mathrm{A}+\mathrm{B})$ GGAACC $\mathrm{CAGG}(-13)$$$
(\mathrm{C}+\mathrm{D}) \text { GGAACC CGGCTAATTG TCAGG }(+1,-2)
$$

FIGURE 4 | Characterization of the $5^{\prime}$ and $3^{\prime}$ junctions of the knock-in at the otx2 locus. Representative gel pictures of the otx2:venus (A) and otx2:tRFP (B) knock-in alleles from one founder (lanes 1 and 3 ) or wild-type siblings (lanes 2 and 4). Lanes 1 and 2 show the $5^{\prime}$ junction PCR (primer pair A+B from Figure 1) and lanes 3 and 4 show the $3^{\prime}$ junction (primers $C+D$ from Figure 1). (C) DNA sequence analysis of the $5^{\prime}$ and $3^{\prime}$ junctions and mutations; "-" denotes deletion and "+" denotes insertion; inserted base pairs are marked in blue. 
thick sections mounted in glycerol, and sections imaged in a Zeiss Axioplan microscope. In situ probe staining matched previously described expression for both otx2 (Mercier et al., 1995) and pax2a (Krauss et al., 1991). For adult zebrafish brain sections, freeze-thawed and air-dried sections were treated with $100 \%$ Methanol (500 $\mu \mathrm{l} / \mathrm{slide})$ for $10 \mathrm{~min}$, washed in PBSTritonX100 (PBS-Tx) buffer prior to incubation with in situ probe (1:100 dilution), and denatured at $70^{\circ} \mathrm{C}$ in hybridization buffer. Hybridization was done overnight at $60^{\circ} \mathrm{C}$ in a humidified chamber. Excess/unbound probe was removed by rigorous washing with $1 \times$ SSC/50\% formamide solution at $62^{\circ} \mathrm{C}$. Sections were then washed with maleic acid buffer containing Tween20 (MABT) at room temperature (RT). Sections were blocked in DIG blocking reagent (Roche) prior to anti-DIG antibody $(1: 2,000)$ incubation overnight at $4^{\circ} \mathrm{C}$ followed by washes with MABT solution to remove excess antibody. Sections were treated with NBT/BCIP diluted in staining buffer (1:17, NTMT) and the reaction was developed at RT until a signal appeared on the sections. Staining reaction was stopped by washing with PBS and slides were mounted with $80 \%$ glycerol. Images were acquired on a Zeiss Apotome using a differential interference contrast (DIC) filter and processed using ZEN Blue (ver. 2.3), Adobe Photoshop, and Adobe Illustrator (ver. CS5 and CS6) software.

\section{Immunohistochemistry and Imaging}

General immunohistochemistry (IHC) procedure was followed as described elsewhere (MacDonald, 1999). For IHC, slides were thawed from $-20^{\circ} \mathrm{C}$, air-dried, and washed twice with $1 \mathrm{x}$ PBS Tx, 10 min each time. Primary antibodies were diluted in PBSTx and incubated overnight at $4{ }^{\circ} \mathrm{C}$ and secondary antibodies were similarly diluted but incubated for $2 \mathrm{~h}$ at RT. The primary antibodies used were: polyclonal chicken anti-GFP (1:2,000, Abcam, Cat.No: ab13970) to detect Venus and monoclonal mouse anti-HuC/D (1:150, Invitrogen, Cat.No: A21271), and corresponding Alexa conjugated (488 or 555), highly crossadsorbed, secondary antibodies (1:750, Invitrogen) were used for detection of primary antibodies. DAPI (4', 6-diamidino-2phenylindole; $1: 3,000)$ was used to stain nuclei. Antigen retrieval for $\mathrm{HuC} / \mathrm{D}$ was performed using $10 \mathrm{mM}$ sodium citrate buffer, $\mathrm{pH} 6.0$, at $85^{\circ} \mathrm{C}$ for $15 \mathrm{~min}$. Immunostained samples were imaged on a laser scanning confocal microscope (Leica-SP5) using objectives 20x (0.7 NA), 40x (0.75 NA), or 63x Water (1.2 NA). Images were processed using Leica LAS X, Adobe Photoshop, and Adobe Illustrator (CS5 and CS6) software.

\section{Live Imaging}

Embryos were treated from 20 to $24 \mathrm{hpf}$ with 1-Pheny 2-thiourea (PTU) to block pigmentation and with MS-222 for anesthesia, mounted on a glass bottom dish (MatTek) in 1\% low melting agarose, and imaged on a Zeiss LSM 780 or Leica SP 5 microscope. Images were analyzed using FIJI (open source software) or Imaris (ver. 7, Bitplane), respective TIFF files generated, and figures assembled in Adobe Photoshop (ver. CS5 or CS6). For timelapse imaging, tissue sections spanning $30 \mu \mathrm{m}$, with a $\mathrm{Z}$ interval

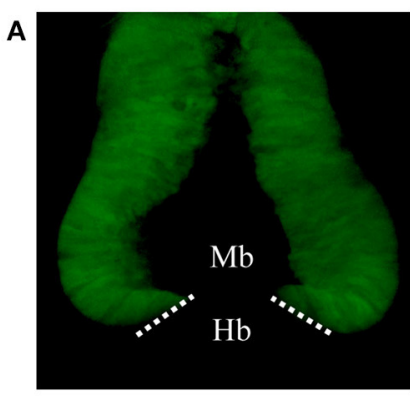

B

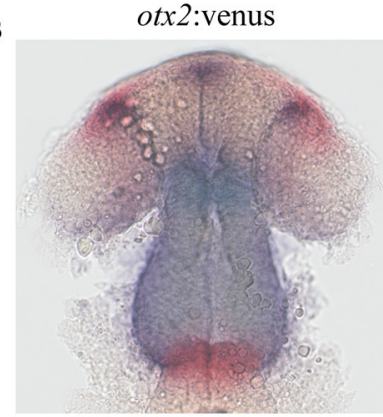

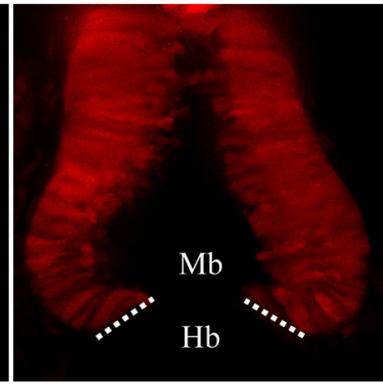

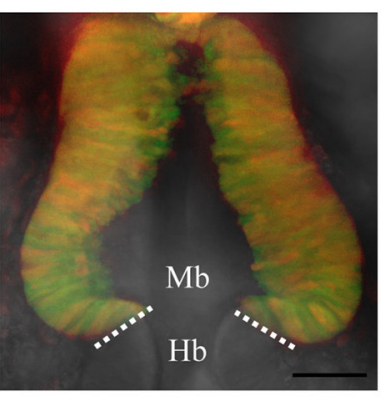

otx $2: \mathrm{tRFP}$

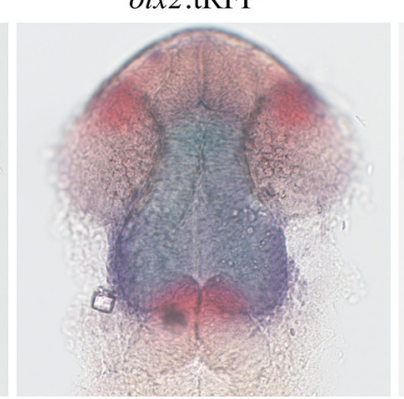

otx2:venus:tRFP

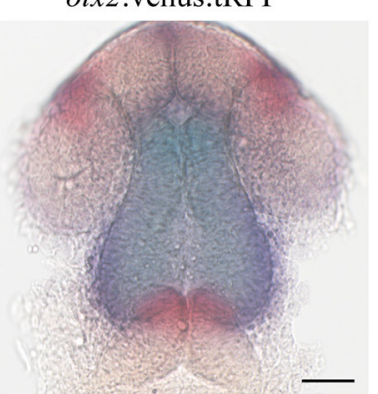

FIGURE 5 | Reporter knock-in into the otx2 locus does not affect endogenous otx2 expression. otx2:venus and otx2:tRFP fish were crossed and sorted into either single (Venus ${ }^{+}$or tRFP $^{+}$) or double positive (Venus ${ }^{+}$and tRFP ${ }^{+}$) embryos at 24 hpf. Images were taken from live embryos anesthetized in MS-222. Panel (A) fluorescent images of the single- and double-positive embryos show no morphological abnormalities. Images are maximum intensity projections covering $50 \mu \mathrm{m}$ tissue with a Z-interval of $2 \mu \mathrm{m}$. Scale bar: $50 \mu \mathrm{m}$. Images were taken from live embryos anesthetized in MS-222. Panel (B) Double in situ hybridization for otx2 (blue) and the MHB marker pax2a (red) show no differences in gene expression pattern or morphology of the MHB region between the single- and double-positive groups. $\mathrm{Mb}$, midbrain; $\mathrm{Hb}$, hindbrain. Scale bar $50 \mu \mathrm{m}$ 
of $2 \mu \mathrm{m}$, were imaged every $8 \mathrm{~min}$ for about $6 \mathrm{~h}$ at $28^{\circ} \mathrm{C}$ on LSM780 (Zeiss) microscopes. Maximum intensity projections of fluorescence and transmitted light images were generated using Imaris (ver. 7, Bitplane).

\section{RESULTS}

\section{Targeted Knock-in at the otx2 Locus to Generate Reporter Lines}

To test if the CRISPR/Cas9-mediated strategy can be used for knocking-in reporters into the zebrafish genome, we chose ot $x 2$ as a candidate gene because, first the caudal limit of the Otx2 marks the MHB, and second, no transgenic ot $x 2$ reporter lines that recapitulate its endogenous expression are currently available (Kurokawa et al., 2006). Fast maturing fluorescent proteins such as Venus and turboRFP (tRFP) were used as reporters. The target site (TS) was selected at about 500 base pairs upstream of the transcription start site of ot $x 2$, and about $1 \mathrm{~kb}$ of bait that included the target region was amplified and cloned in front of the fluorescent reporter (Figure 1, scheme). The target site sequence was verified prior to cloning by DNA sequencing. The efficiency of target-site-cutting was assayed as follows. Both sgRNA and Cas9 were injected into the 1-cell stage embryo, and genomic DNA from individual 24 hpf embryos was isolated for PCR amplification. Loss of the restriction site was used to estimate the efficiency of the sgRNA, and those with $>50 \%$ efficiency were selected (data not shown). Next, the bait plasmid was injected along with the sgRNA and Cas9, and concurrent double strand breaks were generated in the genomic target locus and in the plasmid DNA, resulting in plasmid integration at the target locus, most probably due to the highly active non-homologous end enjoining (NHEJ) repair mechanism of the cells. To identify founders, adult F0 fish were outcrossed with wild-type animals and F1 embryos were screened for fluorescence. Positive embryos and a representative embryo at $24 \mathrm{hfp}$ are shown (Figures 2A,B, 3A,B). Reporter expression perfectly matched the expected expression pattern of Otx2 (Langenberg and Brand, 2005) as a sharp boundary was observed at the MHB, with no ectopic fluorescence in the non-Otx $2^{+}$regions. Two founders were identified for each construct with no difference in reporter gene expression pattern among them (data not shown). The knockin was verified by PCR using primers designed such that the forward primer annealed at the expected knock-in genomic locus (but outside the bait sequence) and the reverse primer within the fluorescent reporter donor plasmid (Figures 4A,B). Sanger DNA sequencing confirmed the knock-in location and showed indels at the $5^{\prime}$ and $3^{\prime}$ integration site (Figure 4C). Germline transmission rates of successful founders were $4 \%$ for ot $x 2$-Venus and $7.6 \%$ for otx2-tRFP reporter lines (summarized in Supplementary Table S1). Currently, ot 22 reporters have been outcrossed for more than six generations and transgene expression has remained stable (data not shown). Further, such outcrossing of the transgenic reporters with wild-type strains over several generations would dilute out any potential off target mutations.

\section{Knock-in Alleles Remain Functional}

Next, we addressed if the knock-in reporters that include a polyA signal and the vector backbone sequences at the $5^{\prime}$ upstream of ATG compromise endogenous gene expression. Thus, we crossed the Venus knock-in fish with the tRFP knockin line and sorted embryos at $24 \mathrm{hpf}$ into either single-positive (Venus $^{+}$or $\mathrm{tRFP}^{+}$) or double-positive (Venus ${ }^{+}$and $\mathrm{tRFP}^{+}$) embryos. Morphological examination showed no differences between the single- and double-positive groups (Figure 5A); subsequent analysis of MHB morphology and double in situ hybridization for ot $x 2$ and the MHB marker pax $2 a$ also showed no differences (Figure 5B). Further, quantitative real time PCR (qRT-PCR) showed no significant difference in ot 2 expression levels in the double positive embryos compared to wild-type (Figure 8D). These data suggest that reporter genes can be efficiently knocked-in at the otx2 locus, and that integration of a reporter plasmid into the non-coding region, upstream of ATG, does not interfere with endogenous gene expression or function.

\section{Practical Application: Live Imaging MHB Development}

Neural tube formation and the underlying cell-biology processes that generate the 3 dimensional structure of the vertebrate brain are of great interest. Hence, real time or time-lapse imaging

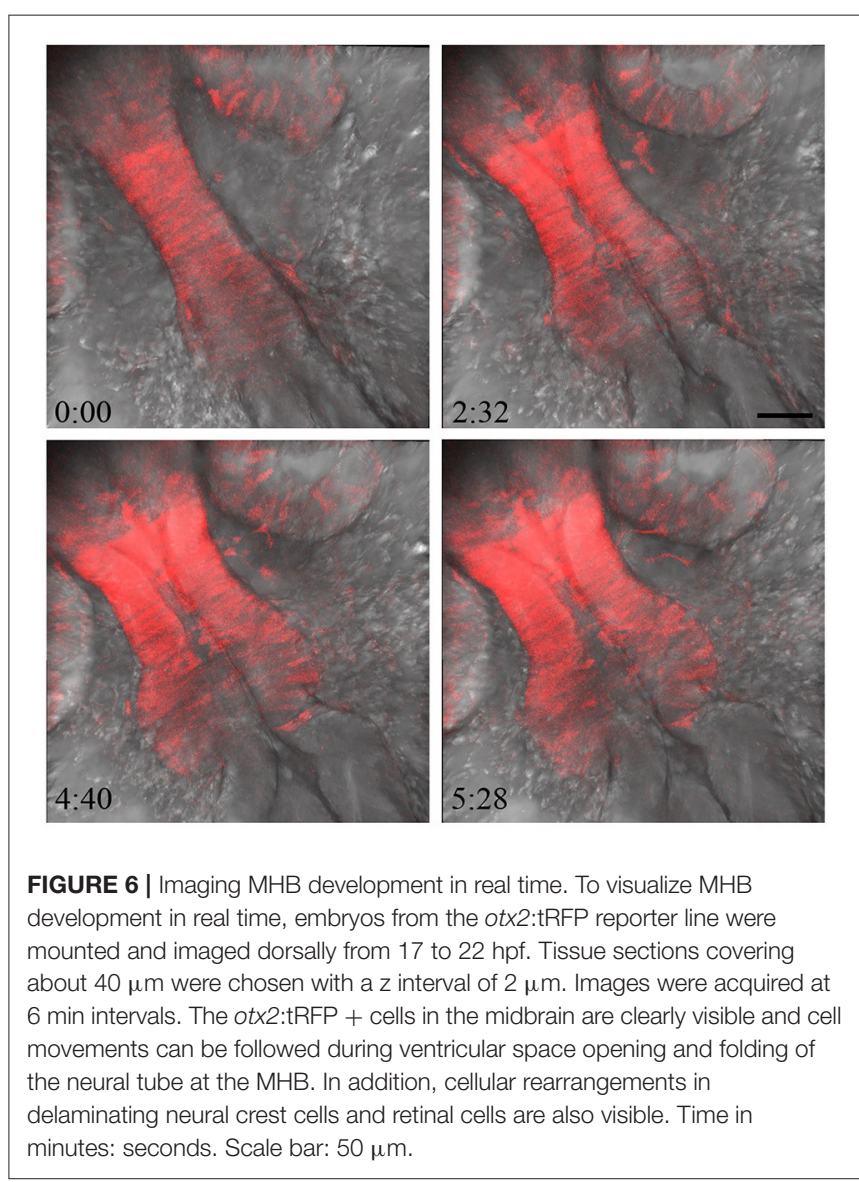




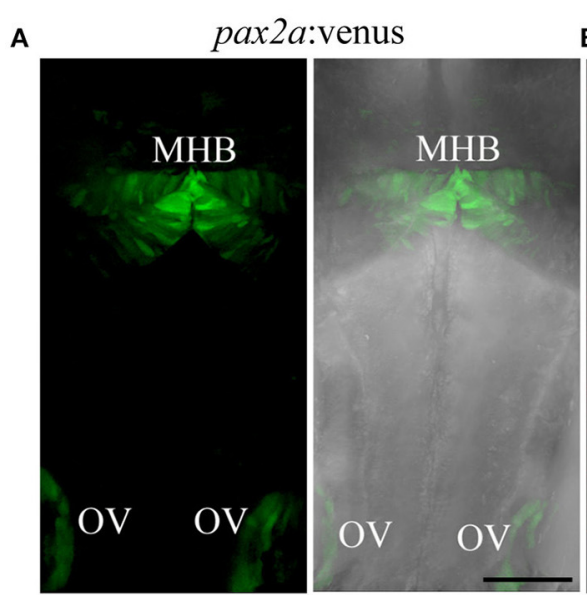

B $\quad \operatorname{pax} 2 a:$ :RFP

C
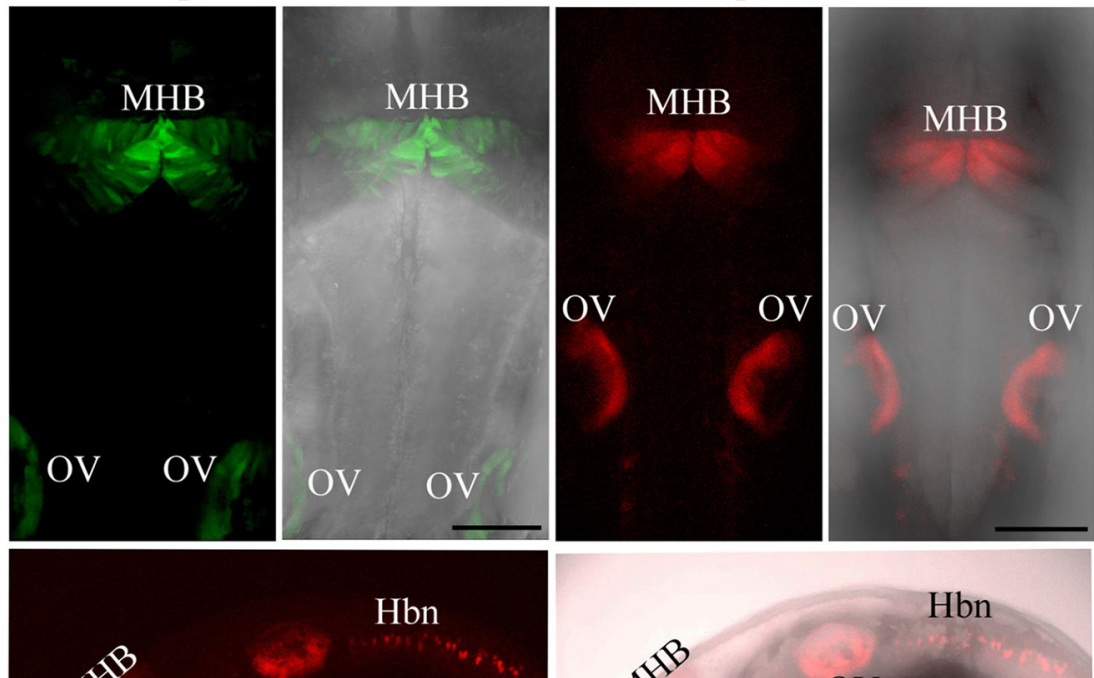

$\mathrm{Hbn}$

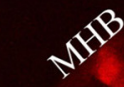

OV

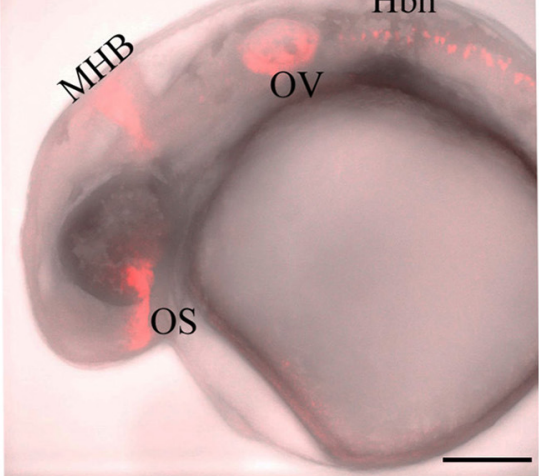

D

pax2a:venus

pax $2 a:$ RFP

pax2a:venus:tRFP
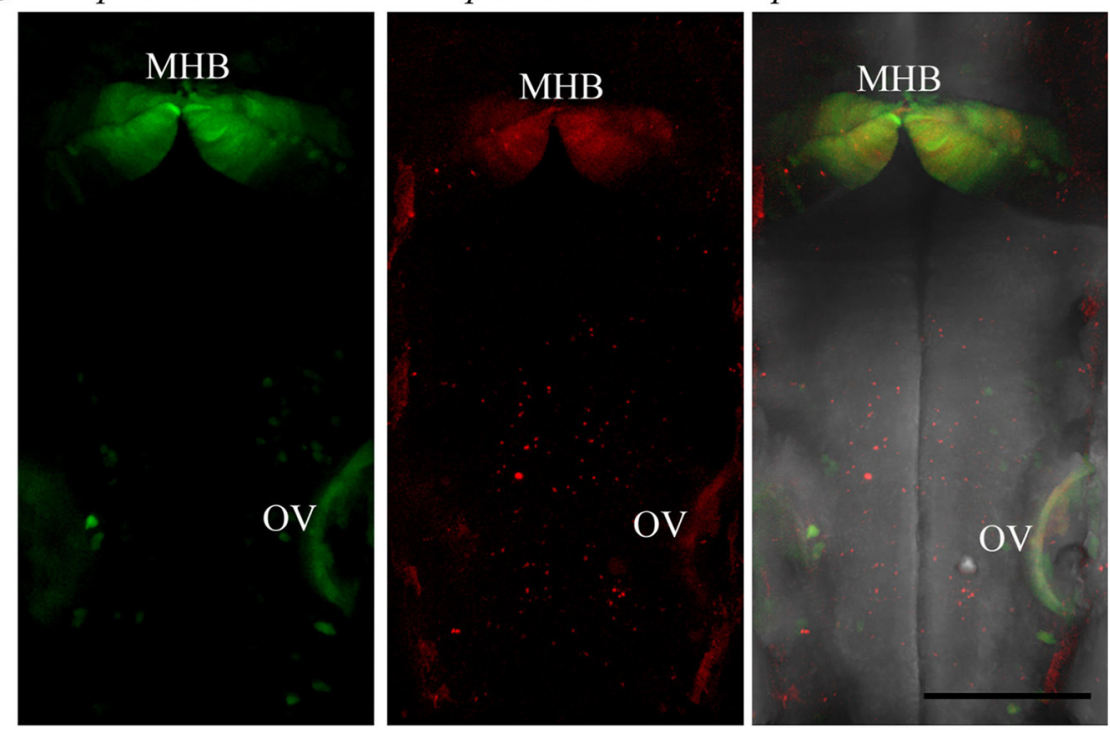

FIGURE 7 | Targeted knock-in of fluorescent reporters into the pax2a locus. Expression of Venus or tRFP driven by the pax2a locus at 24 hpf. Images were taken from live embryos anesthetized in MS-222. (A) Left panel shows dorsal view of Venus expression in the MHB and otic vesicle (OV); right panel shows merged image of fluorescent and transmitted light channels. (B) Left panel shows dorsal view of tRFP expression in the MHB and otic vesicle. (C) Left panel: lateral view of an embryo expressing tRFP in the optic stalk (OS), MHB, OV, and hindbrain neurons (Hbn). Right panel: merged image of fluorescent and transmitted light channels. (D) pax2a:venus and pax2a:tRFP fish were crossed and resulting progeny was sorted into either single $\left(\right.$ Venus $^{+}$or tRFP $\left.^{+}\right)$or double positive $\left(\right.$Venus ${ }^{+}$and tRFP ${ }^{+}$) embryos at $24 \mathrm{hpf}$. Compared to single-positive siblings, double positive embryos show no morphological abnormalities. All images are maximum intensity projections covering $50 \mu \mathrm{m}$ tissue with a Z-interval of $2 \mu \mathrm{m}$. Scale bar $100 \mu \mathrm{m}$. 


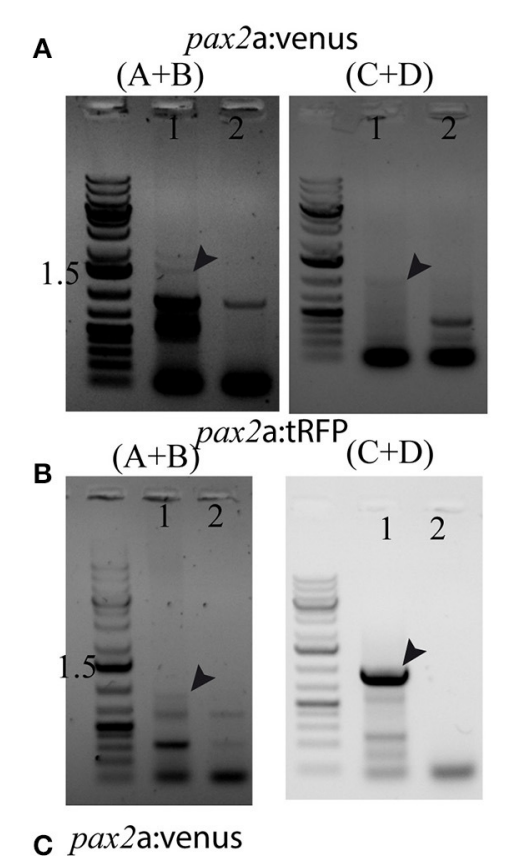

c pax2a:venus

\section{Wt GGGGGGATCTGGGAAGGAGGGGG (A+B) GGGGGGATCTGGGAAG GGGG(-3)

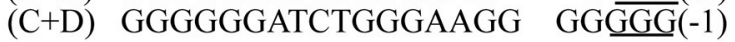 pax2a:tRFP}

\section{Wt GGGGGGATCTGGGAAGGAGGGGG \\ (A+B) GGGGGGATCTGGGAAGG \\ (C+D) GGGGGGATCTGGGAAGGAGGGGG

D

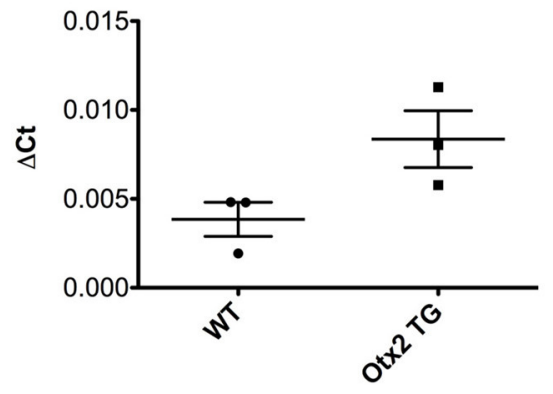

E

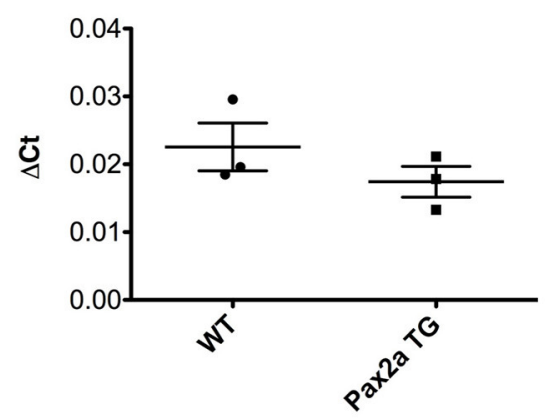

FIGURE 8 | Validation of knock-in insertions at the pax2a locus and gene expression quantification. Representative gel pictures of the pax2a:venus (A) and pax2a:tRFP (B) knock-in alleles from one founder (lane 1) and wild-type siblings (lane 2). Left panel shows $5^{\prime}$ junction PCR (primer pair A+B

(Continued)
FIGURE 8 | Continued

from Figure 1) and the right panel shows $3^{\prime}$ junction (primers $C+D$ from Figure 1). (C) DNA sequence analysis of the $5^{\prime}$ and $3^{\prime}$ junctions and mutations; "-" denotes deletion. (D,E) A comparison of gene expression between homozygous embryos (otx2 or pax2a transgenic animals (TG) positive for both venus and tRFP) and wild-type (WT) embryos (48 hpf) showed no significant differences in the mRNA levels for otx2 (D) and pax2a (E). The two-tailed, unpaired $t$-test was used to calculate statistical significance; each point in the graph represents 1 sample, which contains a pool of 15 embryos. WT vs. otx $2, p=0.072$, WT vs. pax2a, $p=0.288$.

to understand these morphogenetic processes is a valuable approach. We utilized the reporter lines generated using the CRISPR/Cas9 system to observe MHB development in real time. The otx2:tRFP reporter line was used to follow midbrain cells during MHB formation between 17 and $23 \mathrm{hpf}$, when the opening of the ventricular space and constriction at the MHB occur. In addition, otx2:tRFP ${ }^{+}$neural crest cells leaving the neural tube, as well as the otx2:tRFP ${ }^{+}$cells in the retina, can be observed (Figure 6 and Supplementary Movie 1). Consequentially, the ability to co-label cell membranes and nuclei will be valuable in further elucidating the morphogenetic processes that occur during MHB development, including cell shape changes, cell division, and acquisition of apical-basal polarity.

\section{Targeted Knock-in at the pax2a Locus}

To test the knock-in strategy on other genomic loci, we selected the pax $2 a$ locus, as it is one of the earliest MHB markers. Currently, there are no pax $2 a$ reporter lines that recapitulate all its endogenous expression domains (Picker et al., 2002). Again, a highly efficient sgRNA that cleaves the target site upstream of ATG was chosen (data not shown) and bait plasmids were constructed with Venus or tRFP as fluorescent reporters. Embryos were injected and founders were identified as explained above. Fluorescence was evident in the optic stalk, the $\mathrm{MHB}$, and in the optic vesicle at $24 \mathrm{hpf}$ (Figures 7A-C); this expression pattern matches the reported expression pattern for pax2a (Krauss et al., 1991; Lun and Brand, 1998). Germline transmission rates for successful founders were $20 \%$ for Pax $2 a$ :venus and $2.8 \%$ for Pax $2 a$ :tRFP reporters (summarized in Supplementary Table S1). The knock-in was verified by PCR using primers designed such that the forward primer annealed at the expected knock-in genomic locus (but outside the bait sequence) and the reverse primer within the fluorescent reporter donor plasmid (Figures 8A,B). Sanger DNA sequencing confirmed the knock-in location and showed indels at the $5^{\prime}$ and $3^{\prime}$ integration site (Figure 8C). To test if the knockin alleles still expressed endogenous pax $2 a$, we crossed the Venus and tRFP reporter lines and found that the double positive embryos (Venus ${ }^{+}$and $\mathrm{tRFP}^{+}$) were morphologically normal (Figure 7D) and the pax $2 \mathrm{a}$ mRNA remain unchanged in the double positive embryos as quantified by qRT-PCR (Figure 8E). These data suggest that, similar to the otx2 knock-in, the pax $2 a$ locus can also be targeted for genome editing and that the reporter knock-in does not interfere with endogenous gene function. 
otx2:Venus Labels Neurons and the Radial Glia in the Adult Zebrafish Midbrain

We next tested the applicability of the otx2:venus reporter line in adult fish. Anatomically (Figure 9A, schematic), cells expressing Venus were prominently present in the neuronal nuclei or in the neurons of the midbrain, and specifically in the tectum opticum (TeO), the periventricular gray zone of the optic tectum, and the hypothalamus (Figure 9B). Co-expression analysis of Venus, with either HuC/D (a pan-neuronal marker) or $\mathrm{S} 100 \beta$ (a marker for glial cells), revealed that otx2:venus is expressed mostly in $\mathrm{HuC} / \mathrm{D}^{+}$neurons in the gray matter and in glial cells at the ventricular zone of the TeO (Figure 9C). Further,
A

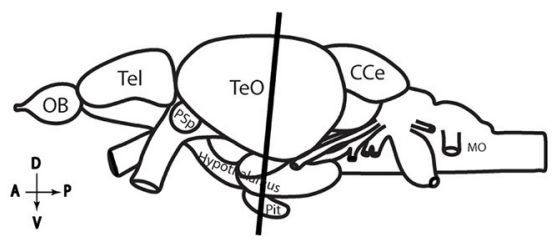

otx2:venus / HuC/D / DAPI otx2:venus

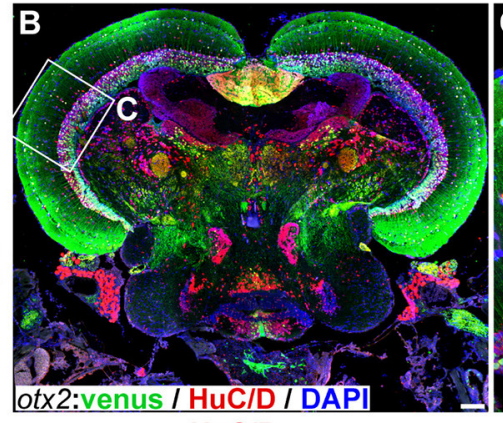

HuC/D

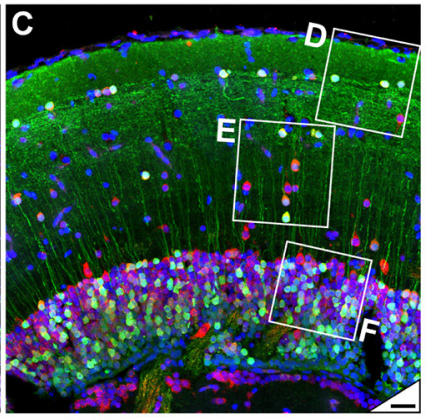

DAPI
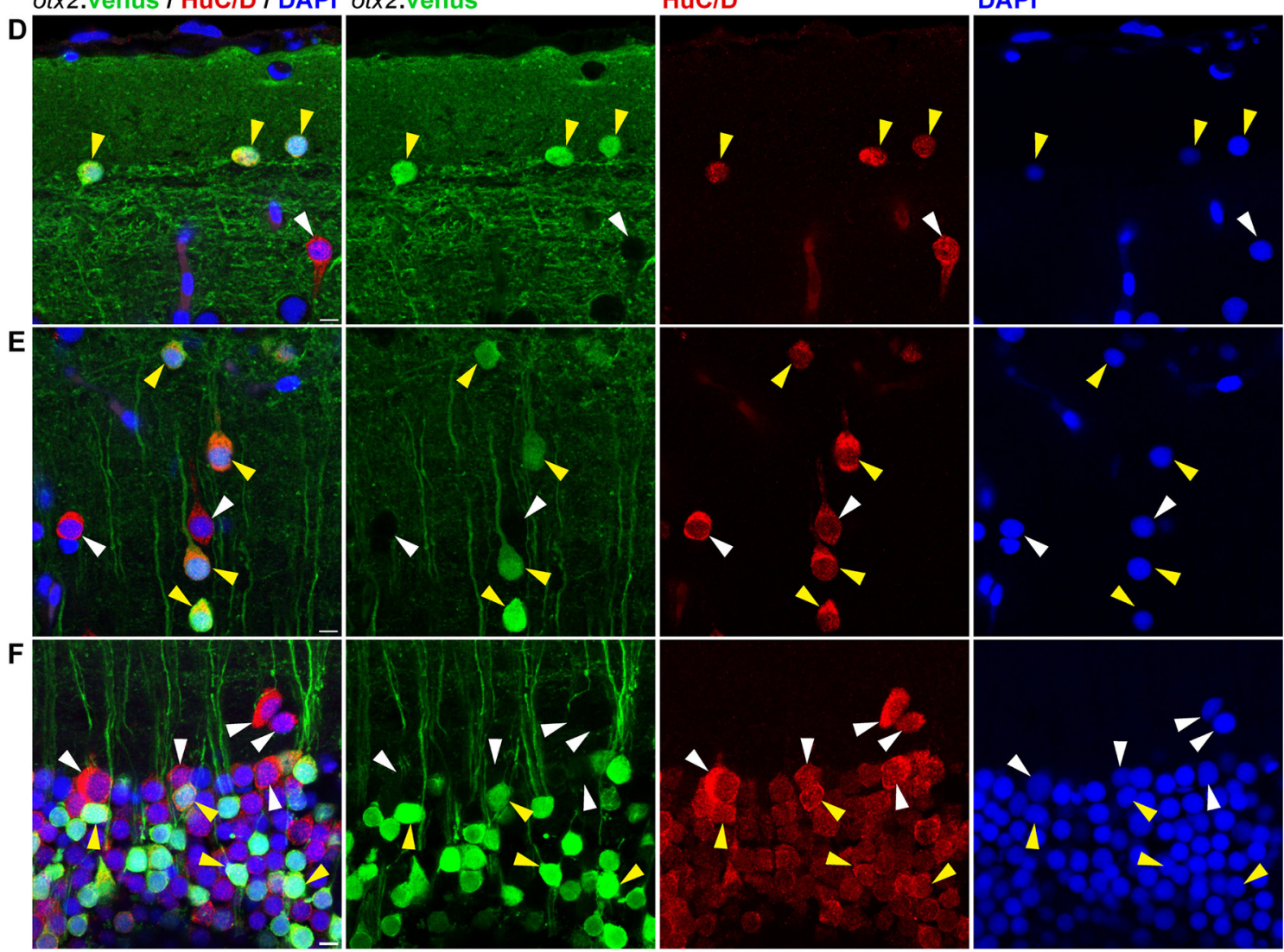

FIGURE 9 | otx2:venus expression in the adult zebrafish midbrain. (A) Schematic representation of the adult zebrafish brain. The position of the cross section of the midbrain with optic tectum, hypothalamus, and pituitary shown in panel (B) is indicated (orientation indicators A->P: anterior to posterior; D->V: dorsal to ventral). (B) Cross section of the midbrain labeled with otx2:venus (green), the pan neuronal marker HuC/D (red) and the nuclear counterstain DAPI (blue). (C) Higher magnification image of the region indicated by white box in panel (B) showing that numerous neurons express otx2:venus. (D-F) Higher magnification images of the regions indicated by white boxes in (C). otx2:venus is co-expressed in HuC/D expressing neurons (yellow arrowheads) in the various tectal sub layers that is characteristic of a teleost midbrain. However, a sub-population of HuC/D positive neurons was negative for otx2:venus (white arrowheads). (D) Type III horizontal neurons that belong to the stratum opticum. (E) Projection neurons that mostly belong to the stratum griseum et album superficiale and centrale regions. (F) Prominent unipolar type XIV interneurons located in the periventricular gray zone of optic tectum. Anatomical descriptions are based on the zebrafish brain atlas (Wullimann et al., 1996), tectal sub layers and neuronal types were interpreted based on Meek (1981) and Meek and Schellart (1978). Scale bars (B): $100 \mu \mathrm{m} ;$ (C): $20 \mu \mathrm{m} ;$ (D-F): $5 \mu \mathrm{m}$. (B,C: maximum intensity projection; D-F: single Z-Plane). 
otx2:venus and $\mathrm{HuC} / \mathrm{D}^{+}$cells were present in various cortical layers of the tectum, such as the stratum opticum (Figure 9D), stratum griseum et album superficiale, and centrale (Figure 9E), and the periventricular gray zone (Figure 9F). However, not all $\mathrm{HuC} / \mathrm{D}^{+}$neurons were labeled by otx2:venus in the tectum, suggesting that Otx2 is expressed only in a sub-population of neurons and that this line might be a good tool to study this specific neuronal network. Based on previously described anatomical distribution and cell shape characteristics (Meek and Schellart, 1978; Meek, 1981), Venus ${ }^{+}$cells appear to be type III horizontal neurons that belong to the stratum opticum (Figure 9D), projection neurons of the cortical layers stratum griseum et album superficiale and centrale (Figure 9E), and XIV interneurons of the periventricular gray zone (Figure 9F). Apart from this, ot $x$ 2:venus also labeled $S 100 \beta^{+}$glial cells in the ventricular zone (anatomical location schematized in Figure 10A, overview images Figures 10B,C). S100 $\beta^{+}$cells are seen at the ventricular zone co-expressing otx2:venus (yellow arrowheads) while the neighboring ot $x 2$ :venus ${ }^{+}$neurons are negative for $S 100 \beta$ (Figure 10D). Most importantly, in the adult midbrain, ot $x$ 2:venus expression mirrored endogenous ot $x 2$ mRNA expression in the various tectal layers, as shown by in situ hybridization (Figures 11A,B). Taken together, otx2:venus CRISPR/Cas9 knock-in line appears to label various neuronal and radial glial populations in the adult zebrafish midbrain. The observed selective labeling presents a useful tool to study specific cells of interest while simultaneously overcoming the potential silencing effects that are often observed with other transgenic approaches.

\section{pax2a:Venus Reporter Expression in the Adult Zebrafish Midbrain}

The pax2a:venus reporter labeled several neuronal subpopulations (anatomical location scheme Figure 12A, overview images Figure 12B). Most importantly, in the adult midbrain, pax2a:venus expression mirrored endogenous pax2a mRNA expression, as shown by in situ hybridization (Figures 12B,C). Essentially, pax2a:venus expression was prominently seen in the neurons of the valvula cerebelli in the hindbrain (red dotted line), the dorsal tegmental nucleus of the midbrain (yellow dotted line area; Figures 12D,E), and other regions of the midbrain. Based on previous anatomical descriptions of the zebrafish brain (Wullimann et al., 1996), pax2a:venus cells could be mapped to neurons present adjacent (left side) to the lateral longitudinal fascicle (yellow dotted circle), with some of these cells possibly belonging to the perilemniscal nucleus (Figures 12F,G). The rostral tegmental nucleus, another neural nucleus, was also positive for pax $2 a$ :venus expression (Figures 12H,I). However, there were no Venus+ cells in the glial domain. These results clearly indicate that pax $2 a$ :venus, similar to otx2:venus, labels a subpopulation of neurons in the mid- and hindbrain in the adult zebrafish.

\section{DISCUSSION}

Here we demonstrate that fluorescent reporters can be efficiently knocked into a specific locus for a gene of interest using the CRISPR/Cas9 system as a genome-editing tool. We have
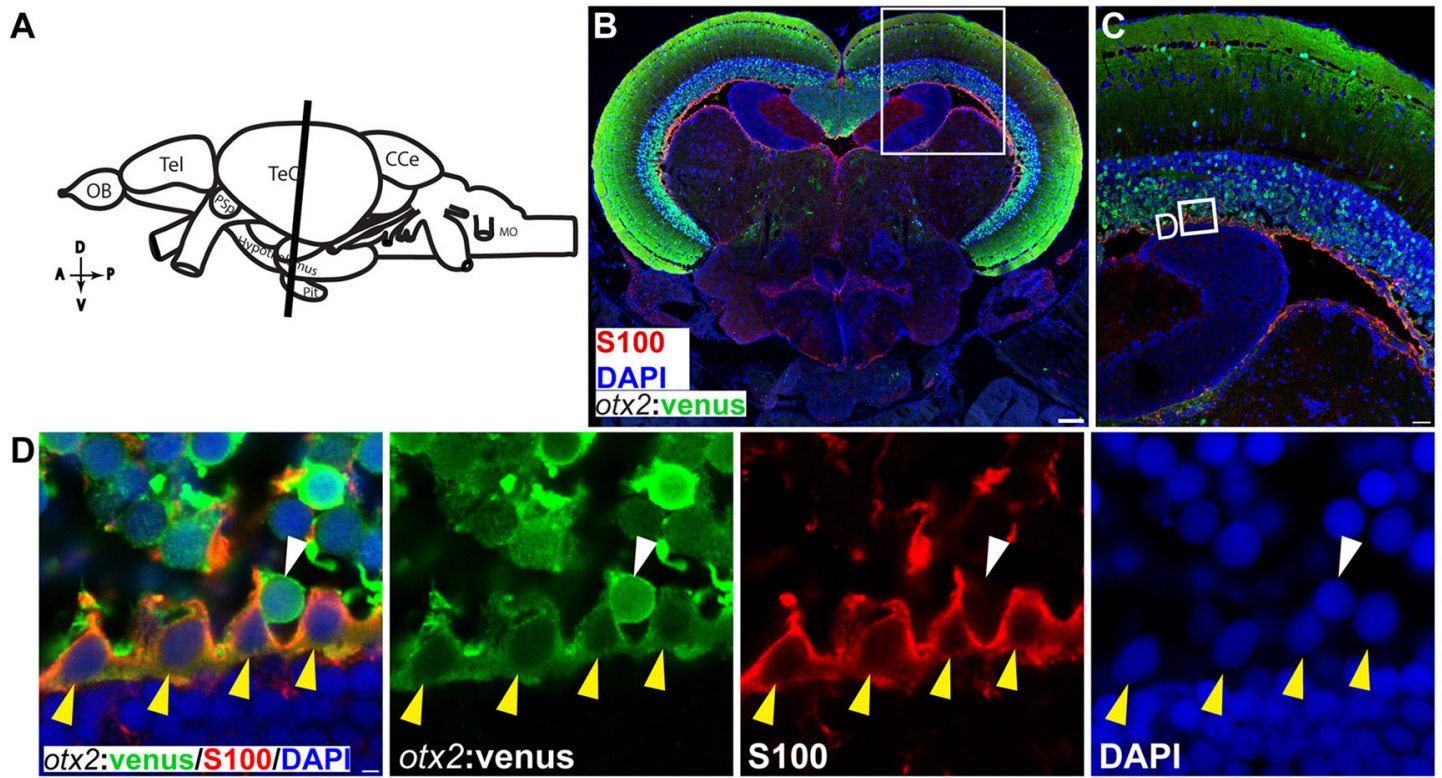

FIGURE 10 | otx2:venus expression in radial glia located at the ventricular zone of the adult zebrafish midbrain. (A) Schematic representation of the adult zebrafish brain. The position of the cross section of the midbrain with optic tectum, hypothalamus, and pituitary shown in panel (B) is indicated (orientation indicators A->P: anterior to posterior; D-> V: dorsal to ventral). (B) Cross section of the midbrain labeled with otx2:venus (green), the radial glial marker S100 (red) and the nuclear counterstain DAPI (blue). (C) Higher magnification of the region from midbrain tectum indicated by white boxes in (B). (D) Higher magnification of an inset from panel (C) showing radial glial cells at the ventricular zone of the midbrain tectum labeled by S100 co-expressing otx2:venus. Anatomical descriptions are based on the zebrafish brain atlas (Wullimann et al., 1996). Scale bars (B): $100 \mu \mathrm{m}$; (C): $25 \mu \mathrm{m}$; (D): 2 (or) $5 \mu \mathrm{m}$. (B,C: maximum intensity projection; D: single Z-plane). 

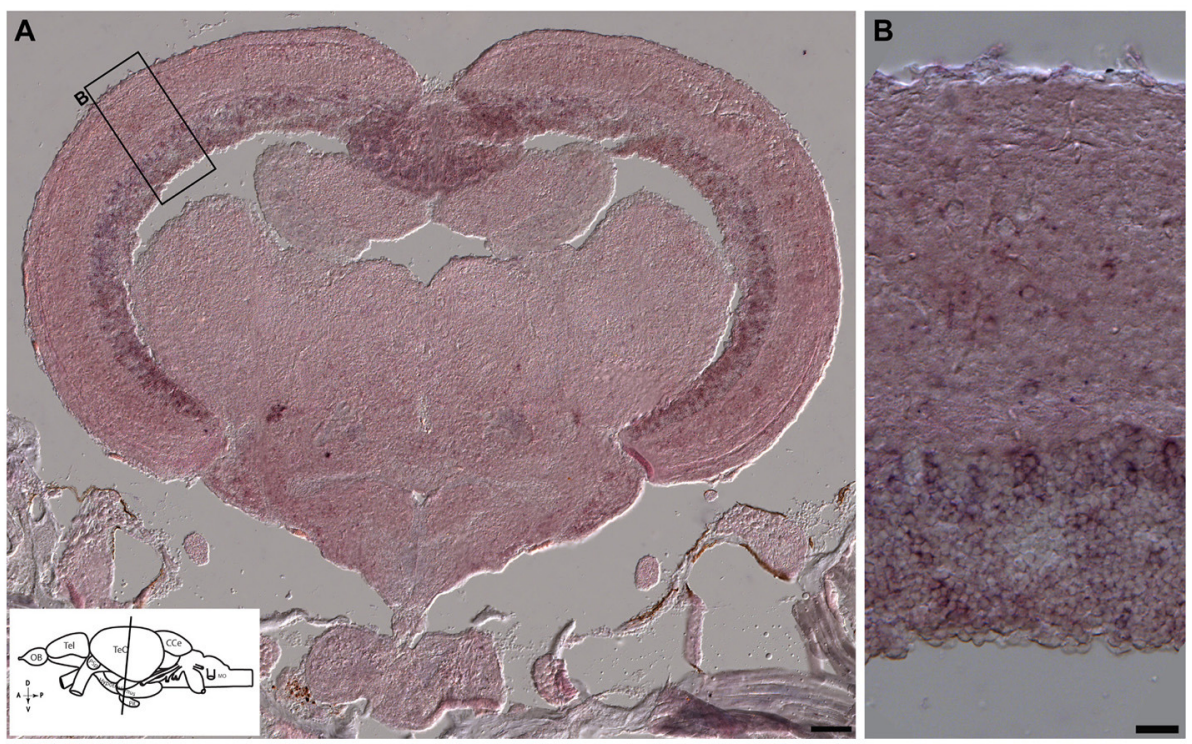

FIGURE 11 | In situ hybridization showing otx2 expression in the adult zebrafish midbrain. (A,B) In situ hybridization for otx2 was carried out on coronal sections of the adult zebrafish midbrain (scheme shown in A). Otx2 signal resembled reporter expression (Figures $\mathbf{8}, \mathbf{9}$ ) in the various tectal layers and particularly strong signals were observed in the periventricular gray zone of the optic tectum. A representative region is the marked with a rectangle and magnified in the adjacent panel $\mathbf{( B )}$. Scale bars (A): $100 \mu \mathrm{m}$; (B): $20 \mu \mathrm{m}$.

generated four knock-in reporter lines targeting two essential genes involved in MHB development, namely otx2 and pax $2 a$. The exact mechanism of integration (knock-in) at the target site has not been explored in this study. However, given that NHEJ has a greater probability of occurrence in zebrafish (Hagmann et al., 1998; Dai et al., 2010) and homologous recombination (HR) of $5 \mathrm{~kb}$ or larger plasmids has not yet been demonstrated, we think that NHEJ is the most probable mechanism of integration. Other studies in zebrafish using similar strategies have also found homology independent mechanisms such as NHEJ to be the primary mechanism that drives integration (Auer et al., 2014; Kimura et al., 2014; Ota et al., 2016).

The combination of methods used here, i.e., CRISPR and non-homologous end joining, has several advantages over BAC or promoter fragment-driven transgenesis. First, by knockingin the reporter just in front of the endogenous ATG, we utilize the native promoter and enhancer elements that regulate endogenous gene expression. Consequently, reporter expression essentially recapitulates native/endogenous promoter activity and gene expression patterns. Indeed, we show that both Venus and tRFP reporter expression precisely match endogenous gene expression of otx 2 and pax $2 a$, both in embryos and adults. The $\operatorname{Tg}(\text { pax } 2 a: G F P)^{e 1 T g}$ transgenic line that we previously generated (Picker et al., 2002) used native promoter/enhancer elements that recapitulated parts of the endogenous pax $2 a$ expression pattern. However, this line also showed ectopic GFP expression in the forebrain, and in rhombomeres 3 and 5 of the hindbrain. Attempts to establish a stable transgenic zebrafish line that recapitulated ot $x 2$ expression using combinations of various promoter/enhancer elements have failed (Kurokawa et al., 2006). Importantly, the knock-in reporter lines reported here also mirror endogenous gene expression patterns in the adult zebrafish brain, as defined by in situ hybridization. This aspect is valuable during both embryonic development and for identifying different neuronal and non-neuronal subtypes in the adult zebrafish brain. To our knowledge, this is the first study on reporter expression for otx 2 and pax $2 a$ in the adult zebrafish brain. Further applications of these reporter lines include their use to better understand the molecular characteristics of Otx $2^{+}$ and $\mathrm{Pax} 2 \mathrm{a}^{+}$cells during different developmental and adult stages by subjecting them to fluorescence activated cell sorting (FACS) for transcriptomic and/or proteomic analyses.

Second, reporter knock-in at the non-coding region, just in front of the ATG did not negatively affect or nullify endogenous gene expression and is consistent with normal MHB morphology in the double transgenic reporters (Venus and tRFP positive). In contrast, recently, Ota et al. (2016) have reported a similar strategy to knock-in eGFP at the same genomic locus for pax $2 a$, but the knocked-in allele generated a pax $2 a$ mutant allele resembling the homozygous pax $2 a$ null allele, no isthmus (noi; Brand et al., 1996). Differences in bait construction might account for why our strategy did not result in null alleles, compared to Ota et al. (2016). Specifically, in our strategy, the plasmid bait supplies the 500 base pairs of the pax $2 a 5^{\prime}$ sequence in front of the ATG, whereas Ota et al. (2016) used a universal bait sequence containing hsp70l promoter/enhancer elements and eGFP. Thus, our knock-in strategy can also be applied for generating Cre- or Gal4-driver lines that can be subsequently used for lineage tracing and loss-of-function studies. Recently, Suzuki et al. have reported the use of mini circles for highly efficient, homology-independent, targeted, in vivo integration. Using this method, they avoid plasmid backbone co-integration 
A

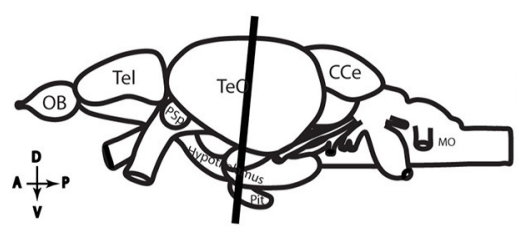

B

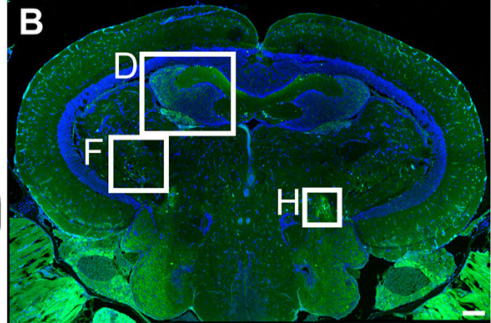

pax2a:venus I DAPI pax2a:venus
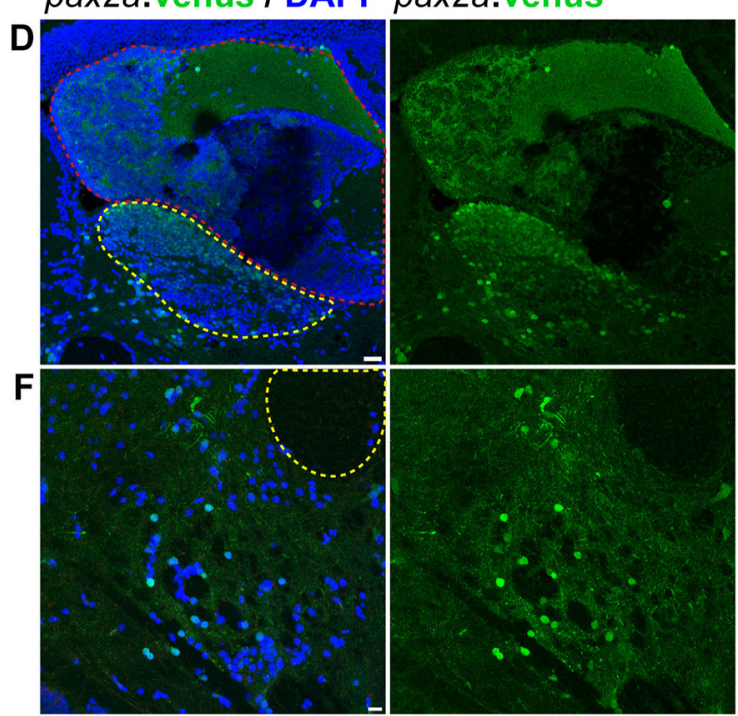

DAPI

$\mathbf{H}$
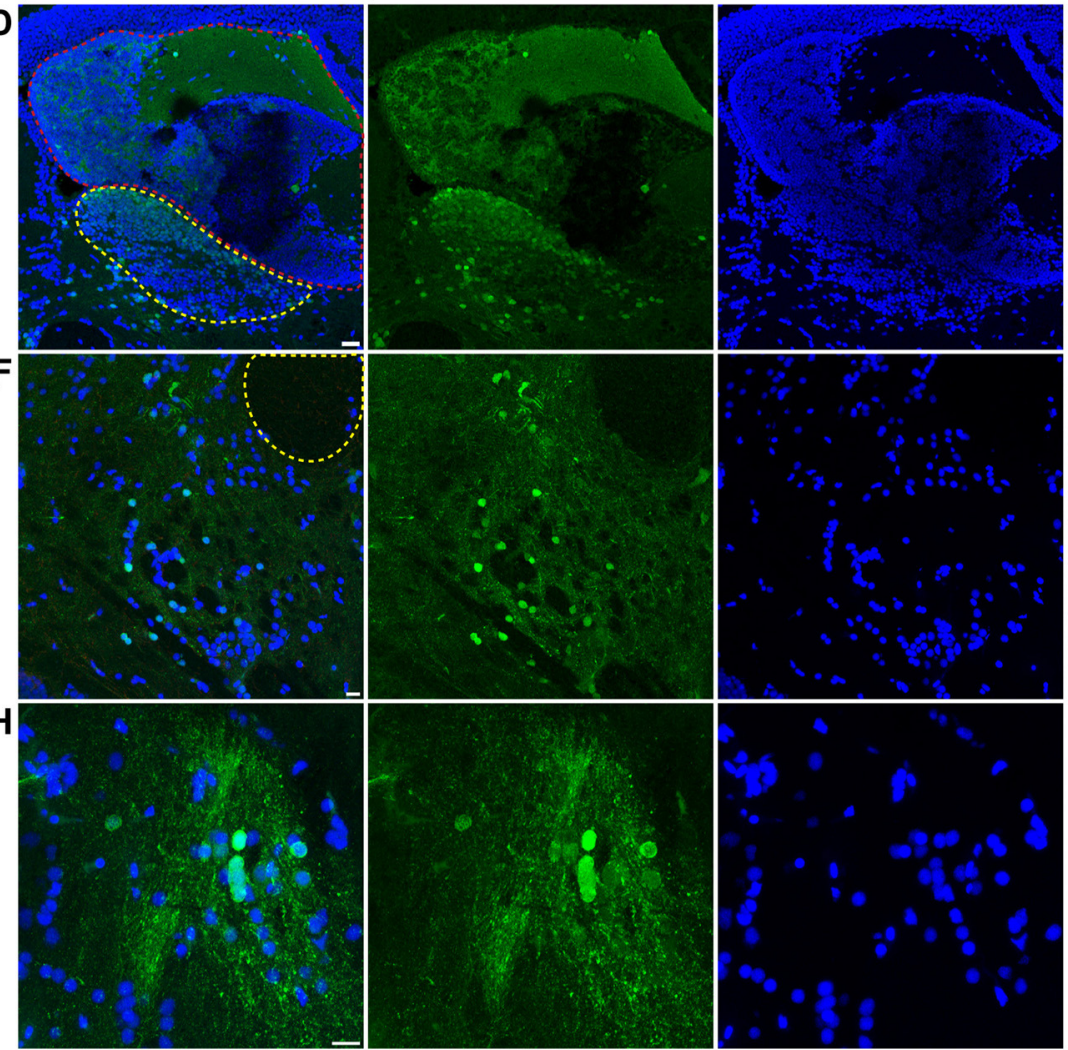

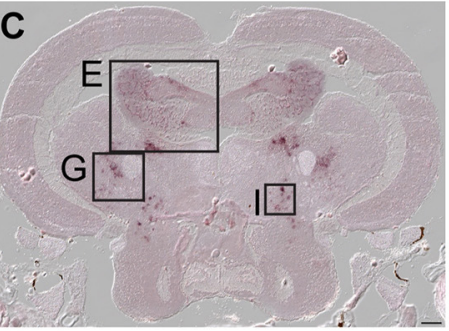

pax2a in situ

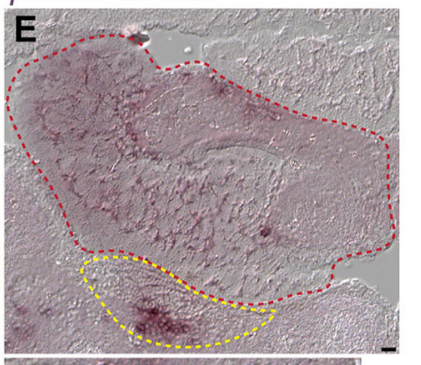

G
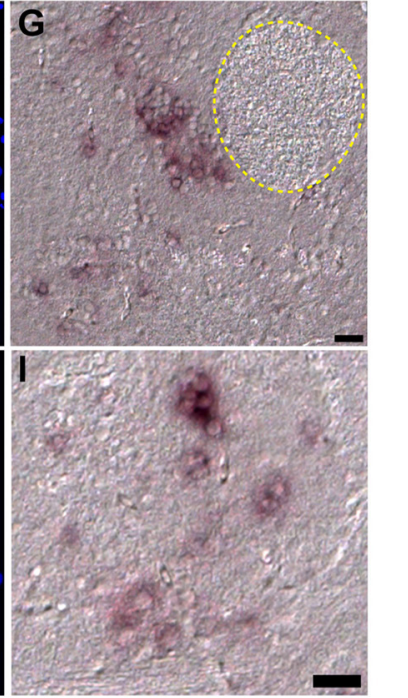

FIGURE 12 | pax2a:venus expression in the adult zebrafish midbrain. (A) Scheme indicating a plane of coronal section of the adult zebrafish mid brain with optic tectum, hypothalamus and the pituitary corresponding to the cross section in "B" (orientation indicators A->P: anterior to posterior; D-> V: Dorsal to Ventral). (B) Cross section of the midbrain immunostained against venus (green) and DAPI (blue). (C) in situ hybridization against pax2a in the midbrain similar to the coronal planes in "A,B" showing similar expression pattern as that of venus in the knock in reporter line. (D,F,H) Higher magnification of a midbrain indicated by white boxes in "B" showing neuronal nuclei expressing venus in regions, valvula cerebelli (red dotted line area) and dorsal tegmental nucleus (yellow dotted line area) "D," neighboring (left side) to the lateral longitudinal fascicle "F" and the rostral tegmental nucleus "H." (E,G,H) Higher magnification of a region from the midbrain tectum indicated by black boxes in "C" showing in situ expression pattern for pax2a showing highly similar pattern to their counter parts expressing pax2a:venus. Anatomical descriptions were based on (zebrafish brain atlas; Wullimann et al., 1996). Scale bars (B,C): $100 \mu \mathrm{m}$; (D,E,G,I): $20 \mu \mathrm{m}$; (F,H): $10 \mu \mathrm{m}$. (B: max intensity projection; $\mathbf{D}, \mathbf{F}, \mathbf{H}$ : single Z-Plane; C,E,G,I: DIC image).

and their design of the targeting construct favors forward integration (Suzuki et al., 2016). The use of mini circles could be easily adapted to improve our strategy as it will avoid plasmid backbone integration, and the smaller size of mini circles may then improve targeting efficiency.

Third, the relatively high germ line transmission rates (ranging from 2.8 to 20\%) will facilitate targeting of several gene loci as potential reporter lines. Hence, easy generation of Cre/Gal4-based driver lines is feasible because the target site resides in the non-coding region, making screening for in-frame insertions unnecessary. It is important to mention here that fluorescent protein expression is indeed a read-out of the promoter activity. It is for this reason that we chose fast folding proteins like Venus and tRFP as reporters, so that positive cells are labeled rapidly after promoter activation. However, one has to note that these fluorescent proteins have a half-life of about $24 \mathrm{~h}$ (Li et al., 1998), and thus, cannot be ideally used to study temporal dynamics; this could be overcome 
by using fast degradable fluorescent proteins. On the other hand, the persistence of fluorescent proteins can also be used advantageously for short-term lineage tracing and assessment of cell fate.

Zebrafish mutants with loss of function phenotypes such as the no isthmus (noi), acerebellar (ace), and spiel-ohne-grenzen $(s p g)$, were identified from large-scale mutagenesis screens and have been fundamental in elucidating the core gene network that regulates MHB formation and maintenance (Brand et al., 1996; Schier et al., 1996; Lun and Brand, 1998; Reifers et al., 1998; Reim and Brand, 2002). The underlying mutations are strong loss of function or null alleles that result in the survival of these mutants for only a few days after birth. Furthermore, very little is known about the expression patterns of the core MHB genes in the larval and adult brains and even less is known about their function under homeostatic and regenerative conditions. Thus, generating CreER driver lines using this knock-in strategy for important players of several gene families involved in $\mathrm{MHB}$ development (Otx, Gbx, Wnt, Fgf8, Pax, Eng) will facilitate loss/gain-of- function studies that can be spatially and temporally tracked. We show that otx2:venus marks some of the neuronal and radial glial cells in the optic tectum and that pax $2 a$ :venus labels neurons in the valvula cerebelli of the hindbrain and other neural nuclei in the midbrain. However, further studies using various marker combinations are required to completely map the cell types that express Otx2 or Pax2.

\section{AUTHOR CONTRIBUTIONS}

GK and MB conceived the project and designed experiments. GK generated the transgenic zebrafish lines and characterized them in embryonic stages, AC generated data on the adult zebrafish brain, and AM generated reagents for in situ hybridization,

\section{REFERENCES}

Adolf, B., Chapouton, P., Lam, C. S., Topp, S., Tannhauser, B., Strahle, U., et al. (2006). Conserved and acquired features of adult neurogenesis in the zebrafish telencephalon. Dev. Biol. 295, 278-293. doi: 10.1016/j.ydbio.2006.03.023

Alunni, A., and Bally-Cuif, L. (2016). A comparative view of regenerative neurogenesis in vertebrates. Development 143, 741-753. doi: $10.1242 /$ dev. 122796

Auer, T. O., Duroure, K., De Cian, A., Concordet, J. P., and Del Bene, F. (2014). Highly efficient CRISPR/Cas9-mediated knock-in in zebrafish by homologyindependent DNA repair. Genome Res. 24, 142-153. doi: 10.1101/gr.1616 38.113

Brand, M., Granato, M., and Nüsslein-Volhard, C. (2002). "Keeping and raising zebrafish," in Zebrafish: A Practical Approach, eds C. Nüsslein-Volhard and R. Dahm (Oxford: Oxford University Press), 7-37.

Brand, M., Heisenberg, C. P., Jiang, Y. J., Beuchle, D., Lun, K., Furutani-Seiki, M., et al. (1996). Mutations in zebrafish genes affecting the formation of the boundary between midbrain and hindbrain. Development 123, 179-190.

Cong, L., Ran, F. A., Cox, D., Lin, S., Barretto, R., Habib, N., et al. (2013). Multiplex genome engineering using CRISPR/Cas systems. Science 339, 819-823. doi: 10.1126/science. 1231143

Dai, J., Cui, X., Zhu, Z., and Hu, W. (2010). Non-homologous end joining plays a key role in transgene concatemer formation in transgenic zebrafish embryos. Int. J. Biol. Sci. 6, 756-768. doi: 10.7150/ijbs.6.756 acquired, and analyzed data from adult brain sections and performed q-RT PCR experiments. GK, AC, and MB wrote the manuscript.

\section{FUNDING}

GK was supported by post-doctoral fellowships from Swedish research council (Vetenskapsrådet) and an EMBO longterm fellowship (ALTF 350-2011). This work was also supported by an ERC advanced grant (Zf-BrainReg) and project grant of the German Research Foundation (Deutsche Forschungsgemeinschaft, project number BR 1746/6-1) to MB.

\section{ACKNOWLEDGMENTS}

We thank the Chen and Wente labs for plasmids for preparing Cas9 and sgRNA mRNA (via addgene), pCS2+ Venus (Andrew Oates lab), and Stefan Hans for comments on the manuscript, past and present members of the Brand lab for discussions, and Vasuprada Iyengar for language and content editing. We thank Marika Fischer, Jitka Michling, Claudia Meyer, and Daniela Mögel for dedicated zebrafish care. The Light Microscopy Facility, a core facility of BIOTEC/CRTD at the Technische Universität Dresden, supported this work.

\section{SUPPLEMENTARY MATERIAL}

The Supplementary Material for this article can be found online at: http://journal.frontiersin.org/article/10.3389/fnana. 2017.00052/full\#supplementary-material

Supplementary Table S1 | Table showing indel efficiency and germ-line transmission efficiency for all the transgenic lines.

Supplementary Table S2 | List of PCR primers.

Dworkin, S., and Jane, S. M. (2013). Novel mechanisms that pattern and shape the midbrain-hindbrain boundary. Cell. Mol. Life Sci. 70, 3365-3374. doi: 10.1007/s00018-012-1240-x

Grandel, H., and Brand, M. (2013). Comparative aspects of adult neural stem cell activity in vertebrates. Dev. Genes Evol. 223, 131-147. doi: 10.1007/s00427-012-0425-5

Grandel, H., Kaslin, J., Ganz, J., Wenzel, I., and Brand, M. (2006). Neural stem cells and neurogenesis in the adult zebrafish brain: origin, proliferation dynamics, migration and cell fate. Dev. Biol. 295, 263-277. doi: 10.1016/j.ydbio.2006.03.040

Hagmann, M., Bruggmann, R., Xue, L., Georgiev, O., Schaffner, W., Rungger, D., et al. (1998). Homologous recombination and DNA-end joining reactions in zygotes and early embryos of zebrafish (Danio rerio) and Drosophila melanogaster. Biol. Chem. 379, 673-681. doi: 10.1515/bchm.1998.379. 6.673

Hoshijima, K., Jurynec, M. J., and Grunwald, D. J. (2016). Precise editing of the zebrafish genome made simple and efficient. Dev. Cell 36, 654-667. doi: 10.1016/j.devcel.2016.02.015

Jao, L. E., Wente, S. R., and Chen, W. (2013). Efficient multiplex biallelic zebrafish genome editing using a CRISPR nuclease system. Proc. Natl. Acad. Sci. U.S.A. 110, 13904-13909. doi: 10.1073/pnas.1308335110

Kimmel, C. B., Ballard, W. W., Kimmel, S. R., Ullmann, B., and Schilling, T. F. (1995). Stages of embryonic development of the zebrafish. Dev. Dyn. 203, 253-310. doi: 10.1002/aja.1002030302 
Kimura, Y., Hisano, Y., Kawahara, A., and Higashijima, S. (2014). Efficient generation of knock-in transgenic zebrafish carrying reporter/driver genes by CRISPR/Cas9-mediated genome engineering. Sci. Rep. 4:6545. doi: $10.1038 /$ srep06545

Kizil, C., Kaslin, J., Kroehne, V., and Brand, M. (2012). Adult neurogenesis and brain regeneration in zebrafish. Dev. Neurobiol. 72, 429-461. doi: 10.1002/dneu.20918

Krauss, S., Johansen, T., Korzh, V., and Fjose, A. (1991). Expression pattern of zebrafish pax genes suggests a role in early brain regionalization. Nature 353, 267-270. doi: 10.1038/353267a0

Kurokawa, D., Sakurai, Y., Inoue, A., Nakayama, R., Takasaki, N., Suda, Y., et al. (2006). Evolutionary constraint on Otx2 neuroectoderm enhancers-deep conservation from skate to mouse and unique divergence in teleost. Proc. Natl. Acad. Sci. U.S.A. 103, 19350-19355. doi: 10.1073/pnas.0604686103

Langenberg, T., and Brand, M. (2005). Lineage restriction maintains a stable organizer cell population at the zebrafish midbrain-hindbrain boundary. Development 132, 3209-3216. doi: 10.1242/dev.01862

Li, X., Zhao, X., Fang, Y., Jiang, X., Duong, T., Fan, C., et al. (1998). Generation of destabilized green fluorescent protein as a transcription reporter. J. Biol. Chem. 273, 34970-34975. doi: 10.1074/jbc.273.52.34970

Livak, K. J., and Schmittgen, T. D. (2001). Analysis of relative gene expression data using real-time quantitative PCR and the 2(-Delta Delta C(T)) Method. Methods 25, 402-408. doi: 10.1006/meth.2001.1262

Lun, K., and Brand, M. (1998). A series of no isthmus (noi) alleles of the zebrafish pax2.1 gene reveals multiple signaling events in development of the midbrain-hindbrain boundary. Development 125, 3049-3062.

MacDonald, R. (1999). Zebrafish immunohistochemistry. Methods Mol. Biol. 127, 77-88. doi: 10.1385/1-59259-678-9:77

Martinez-Barbera, J. P., Signore, M., Boyl, P. P., Puelles, E., Acampora, D., Gogoi, R., et al. (2001). Regionalisation of anterior neuroectoderm and its competence in responding to forebrain and midbrain inducing activities depend on mutual antagonism between OTX2 and GBX2. Development 128, 4789-4800.

Meek, J. (1981). A golgi-electron microscopic study of goldfish optic tectum. I. description of afferents, cell types, and synapses. J. Comp. Neurol. 199, 149-173. doi: $10.1002 /$ cne.901990202

Meek, J., and Schellart, N. A. M. (1978). A golgi study of goldfish optic tectum. J. Comp. Neurol. 182, 89-121. doi: 10.1002/cne.901820107

Mercier, P., Simeone, A., Cotelli, F., and Boncinelli, E. (1995). Expression pattern of two otx genes suggests a role in specifying anterior body structures in zebrafish. Int. J. Dev. Biol. 39, 559-573.

Montague, T. G., Cruz, J. M., Gagnon, J. A., Church, G. M., and Valen, E. (2014). CHOPCHOP: a CRISPR/Cas9 and TALEN web tool for genome editing. Nucleic Acids Res. 42, W401-W407. doi: 10.1093/nar/gku410

Muto, A., Ohkura, M., Abe, G., Nakai, J., and Kawakami, K. (2013). Real-time visualization of neuronal activity during perception. Curr. Biol. 23, 307-311. doi: 10.1016/j.cub.2012.12.040

Ota, S., Taimatsu, K., Yanagi, K., Namiki, T., Ohga, R., Higashijima, S. I., et al. (2016). Functional visualization and disruption of targeted genes using CRISPR/Cas9-mediated eGFP reporter integration in zebrafish. Sci. Rep. 6:34991. doi: 10.1038/srep34991

Picker, A., Scholpp, S., Bohli, H., Takeda, H., and Brand, M. (2002). A novel positive transcriptional feedback loop in midbrain-hindbrain boundary development is revealed through analysis of the zebrafish pax 2.1 promoter in transgenic lines. Development 129, 3227-3239.

Raible, F., and Brand, M. (2004). Divide et Impera-the midbrainhindbrain boundary and its organizer. Trends Neurosci. 27, 727-734. doi: 10.1016/j.tins.2004.10.003
Reifers, F., Bohli, H., Walsh, E. C., Crossley, P. H., Stainier, D. Y., and Brand, M. (1998). Fgf8 is mutated in zebrafish acerebellar (ace) mutants and is required for maintenance of midbrain-hindbrain boundary development and somitogenesis. Development 125, 2381-2395.

Reim, G., and Brand, M. (2002). Spiel-ohne-grenzen/pou2 mediates regional competence to respond to Fgf8 during zebrafish early neural development. Development 129, 917-933.

Rhinn, M., and Brand, M. (2001). The midbrain-hindbrain boundary organizer. Curr. Opin. Neurobiol. 11, 34-42. doi: 10.1016/S0959-4388(00)00171-9

Rhinn, M., Lun, K., Amores, A., Yan, Y. L., Postlethwait, J. H., and Brand, M. (2003). Cloning, expression and relationship of zebrafish gbx1 and gbx2 genes to Fgf signaling. Mech. Dev. 120, 919-936. doi: 10.1016/S0925-4773(03) 00135-7

Rhinn, M., Picker, A., and Brand, M. (2006). Global and local mechanisms of forebrain and midbrain patterning. Curr. Opin. Neurobiol. 16, 5-12. doi: 10.1016/j.conb.2006.01.005

Robles, E., Smith, S. J., and Baier, H. (2011). Characterization of genetically targeted neuron types in the zebrafish optic tectum. Front. Neural Circuits 5:1. doi: 10.3389/fncir.2011.00001

Schier, A. F., Neuhauss, S. C., Harvey, M., Malicki, J., Solnica-Krezel, L., Stainier, D. Y., et al. (1996). Mutations affecting the development of the embryonic zebrafish brain. Development 123, 165-178.

Scott, E. K., and Baier, H. (2009). The cellular architecture of the larval zebrafish tectum, as revealed by Gal4 enhancer trap lines. Front. Neural Circuits 3:13. doi: 10.3389/neuro.04.013.2009

Sunmonu, N. A., Li, K., Guo, Q., and Li, J. Y. (2011). Gbx2 and Fgf8 are sequentially required for formation of the midbrain-hindbrain compartment boundary. Development 138, 725-734. doi: 10.1242/dev.055665

Suzuki, K., Tsunekawa, Y., Hernandez-Benitez, R., Wu, J., Zhu, J., Kim, E. J., et al. (2016). In vivo genome editing via CRISPR/Cas9 mediated homology-independent targeted integration. Nature 540, 144-149. doi: 10.1038/nature20565

Tossell, K., Kiecker, C., Wizenmann, A., Lang, E., and Irving, C. (2011). Notch signalling stabilises boundary formation at the midbrain-hindbrain organiser. Development 138, 3745-3757. doi: 10.1242/dev.070318

Westerfield, M. (2000). The Zebrafish Book. A Guide for the Laboratory Use of Zebrafish (Danio rerio), 4th Edn. Eugene, OR: University of Oregon Press.

Wullimann, M. F., Rupp, B., and Reichert, H. (eds.). (1996). Neuroanatomy of the Zebrafish Brain. A Topological Atlas. Basel: Birkhäuser Verlag. doi: 10.1007/978-3-0348-8979-7

Wurst, W., and Bally-Cuif, L. (2001). Neural plate patterning: upstream and downstream of the isthmic organizer. Nat. Rev. Neurosci. 2, 99-108. doi: $10.1038 / 35053516$

Zervas, M., Millet, S., Ahn, S., and Joyner, A. L. (2004). Cell behaviors and genetic lineages of the mesencephalon and rhombomere 1 . Neuron 43, 345-357. doi: 10.1016/j.neuron.2004.07.010

Conflict of Interest Statement: The authors declare that the research was conducted in the absence of any commercial or financial relationships that could be construed as a potential conflict of interest.

Copyright (c) 2017 Kesavan, Chekuru, Machate and Brand. This is an open-access article distributed under the terms of the Creative Commons Attribution License (CC $B Y)$. The use, distribution or reproduction in other forums is permitted, provided the original author(s) or licensor are credited and that the original publication in this journal is cited, in accordance with accepted academic practice. No use, distribution or reproduction is permitted which does not comply with these terms. 